\title{
Alkaptonuria with extensive ochronotic degeneration of the Achilles tendon and its surgical treatment: a case report and literature review
}

\author{
Nesrin Mwafi ${ }^{1, * \odot}$, Ali Alasmar ${ }^{2 \odot}$, Monther Al-Momani ${ }^{3 \odot}$, Sattam Alazaydeh ${ }^{4 \odot}$, Omar Alajoulin ${ }^{4}$, \\ Mohammad Alsalem ${ }^{5}$, Heba Kalbouneh ${ }^{5 \odot}$
}

Abstract

Background: Alkaptonuria is a rare genetic metabolic disorder due to deficiency of homogentisate 1,2-dioxygenase (HGD), an enzyme catalyzing the conversion of homogentisate to 4-maleylacetoacetate in the pathway for the catabolism of phenylalanine and tyrosine. HGD deficiency results in accumulation of homogentisic acid and its pigmented polymer. Ochronosis is a bluish-black discoloration due to the deposition of the polymer in collagenous tissues. Extensive ochronotic involvement of the Achilles tendon in alkaptonuria and its surgical treatment is rarely reported.

Case report: A 43-year-old man presented to our clinic in March 2019 with sudden onset of left Achilles tendon pain with no history of prior trauma. Surgical exploration revealed a complete disruption of the tendon at its attachment to the calcaneus. Black pigmentation was extensive and reached the calcaneal tuberosity, extending about $7 \mathrm{~cm}$ from the insertion.

Discussion: Achilles reconstruction was performed using flexor hallucis longus tendon transfer. The patient experienced uncomplicated healing with satisfactory functional results.

Conclusion: Orthopedic surgeons should be aware of the progressive nature of alkaptonuria. Extensive degenerative changes of the ruptured tendon should be suspected so that physicians can plan tendon repair and facilitate prompt surgical intervention.

Keywords: Achilles tendon; alkaptonuria; homogentisic acid; ochronosis; rupture

Alkaptonuria (Online Mendelian Inheritance in Man entry No. 203500) is a rare genetic disorder caused by mutation of the gene for homogentisate 1,2-dioxygenase (EC 1.13.11.5) $(H G D)$, which encodes an enzyme essential for the catabolism of phenylalanine and tyrosine [1]. This deficiency results in accumulation of homogentisic acid (HGA), which polymerizes and forms a dark pigment [2]. The excess HGA and its polymer bind to the connective tissues, leading to their

*Correspondence to: Nesrin Mwafi, Department of Biochemistry and Molecular Biology, Faculty of Medicine, Mutah University, Alkarak 61710, Jordan, email: drnesrin@mutah.edu.jo

${ }^{1}$ Department of Biochemistry and Molecular Biology, Faculty of Medicine, Mutah University, Alkarak 61710, Jordan 2Department of Urology, Prince Hussein Urology Center, Jordanian Royal Medical Services, Amman 11855, Jordan ${ }^{3}$ Department of Radiology, Jordanian Royal Medical Services, Amman 11855, Jordan

${ }^{4}$ Department of Orthopedics and Trauma, Jordanian Royal Medical Services, Amman 11855, Jordan

${ }^{5}$ Department of Anatomy and Histology, School of Medicine, The University of Jordan, Amman 11942, Jordan 
bluish-black pigmentation over the years, a phenomenon known as ochronosis [3]. Alkaptonuria occurs worldwide with a global prevalence of 1:250,000, but the prevalence is higher in certain regions such as Slovakia [4], the Dominican Republic, and Jordan [5, 6]. Patients with undiagnosed alkaptonuria routinely present to orthopedic clinics complaining of low back pain, morning stiffness, and pain in the large joints such as knees and shoulders $[7,8]$.

One of the earliest signs of alkaptonuria is urine that turns black or dark brown upon standing for $24 \mathrm{~h}$ or after exposure to air [5]. Other presentations of alkaptonuria are bluish discoloration of the ear auricle, black cerumen, dark spots in the eye sclera, staining of the teeth, blue speckled discoloration of the skin, rupture of Achilles tendon, and renal and prostatic stones [9-11]. Rupture of the Achilles tendon is reported in the literature as a rare clinical complication usually occurring in male patients with alkaptonuria $[12,13]$. To our knowledge, the first case was reported in 2003 [14]. However, very few cases have been reported in female patients with alkaptonuria $[15,16]$, and the majority of the articles describe an isolated tendon rupture.

As distinct from previous cases, we report a case of alkaptonuria in a patient with nontraumatic Achilles tendon rupture from its bony attachment with the ochronotic pigmentation extending into the calcaneal tuberosity. However, the extensive ochronosis of the tendon and its bony insertion in our patient necessitated the excision of a large tendon segment, leaving a gap similar to that observed in chronic Achilles tendon rupture. To our knowledge, the best method for repair of tendon rupture with a large gap in patients with alkaptonuria is not well reported and has remained unclear.

\section{Case presentation}

A 43-year-old man living in southern Jordan presented to our clinic in March 2019 with sudden onset of left Achilles tendon pain with no history of prior trauma. He had developed progressive low back and knee pain for the previous 5 years, which was managed using nonsteroidal anti-inflammatory drugs. He was diagnosed with alkaptonuria 10 years earlier (urine analysis and biochemical testing confirmed the HGAuria at the time of diagnosis). The patient had discoloration of his teeth, ear pinna, and sclera of the eye (Figure 1), and his urine has been dark since childhood (Figures 2C, D). His sister and brother have been also diagnosed with alkaptonuria, and his parents are related (consanguineous marriage). Genetic analysis identified a homozygous missense variant c. $365 \mathrm{C}>\mathrm{T}$ lies in exon 6 of $H G D$ resulting in the substitution of the amino acid alanine (Ala) with valine (Val) Ala122Val (SNP ID rs544956641) [17] (Figures 2A, B) as deposited in the HGD mutation database [4].

Physical examination revealed a palpable defect over the left Achilles tendon at its insertion. Absence of plantarflexion was observed using a Simmonds-Thompson test. Lumbosacral computed tomography (CT) showed diffuse degenerative changes of the spine (Figure 3). Magnetic resonance imaging (MRI) revealed that the tendinous insertion of the Achilles tendon into the calcaneus tuberosity was completely disrupted with retraction of the proximal part (Figure 4). Surgical exploration through the medial incision to the Achilles tendon down to its insertion site revealed a complete disruption of the tendon at its attachment to calcaneus with fragile, black deposited frayed edges (Figure 5A). The
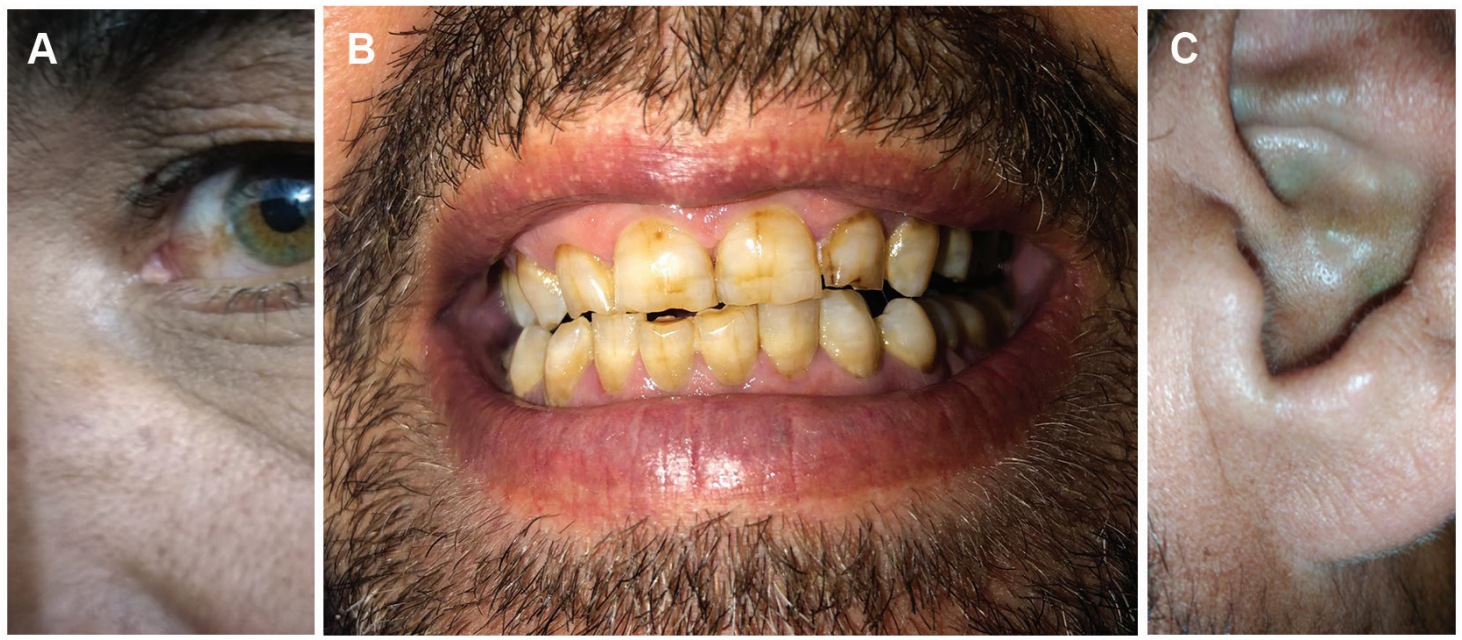

Figure 1. Clinical manifestations of alkaptonuria presented in the reported case. (A) Brownish discoloration of the eye sclera, (B) discoloration of the teeth, (C) bluish discoloration of the ear pinna. With documented consent for publication from the patient. 

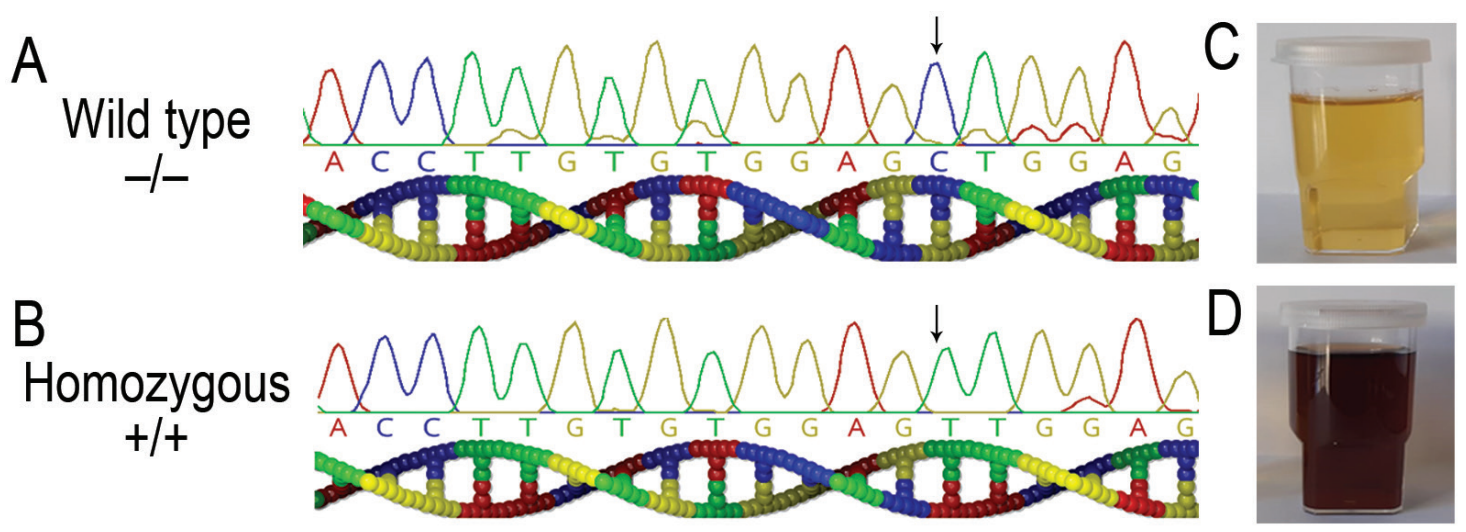

Figure 2. Sanger sequencing analysis of DNA from the patient with the case of alkaptonuria reported here. (A) Electropherogram of exon a6 HGD from a control (wild type) showing $C$ at position 365. (B) Electropherogram of exon a6 HGD from the patient with alkaptonuria demonstrated a point mutation leading to replacement of $C$ with T at position 365 (c.365C $>$ T, p.Ala122Val). The black arrow refers to the position of the missense mutation. (C) Urine sample of control (wild type) did not show any change in color after $24 \mathrm{~h}$. (D) Urine sample from the patient with alkaptonuria in which the color changed from yellow to dark brown or black upon standing for $24 \mathrm{~h}$ due to oxidation of HGA to benzoquinone acetic acid. HGD, homogentisate 1,2-dioxygenase; HGA, homogentisic acid.
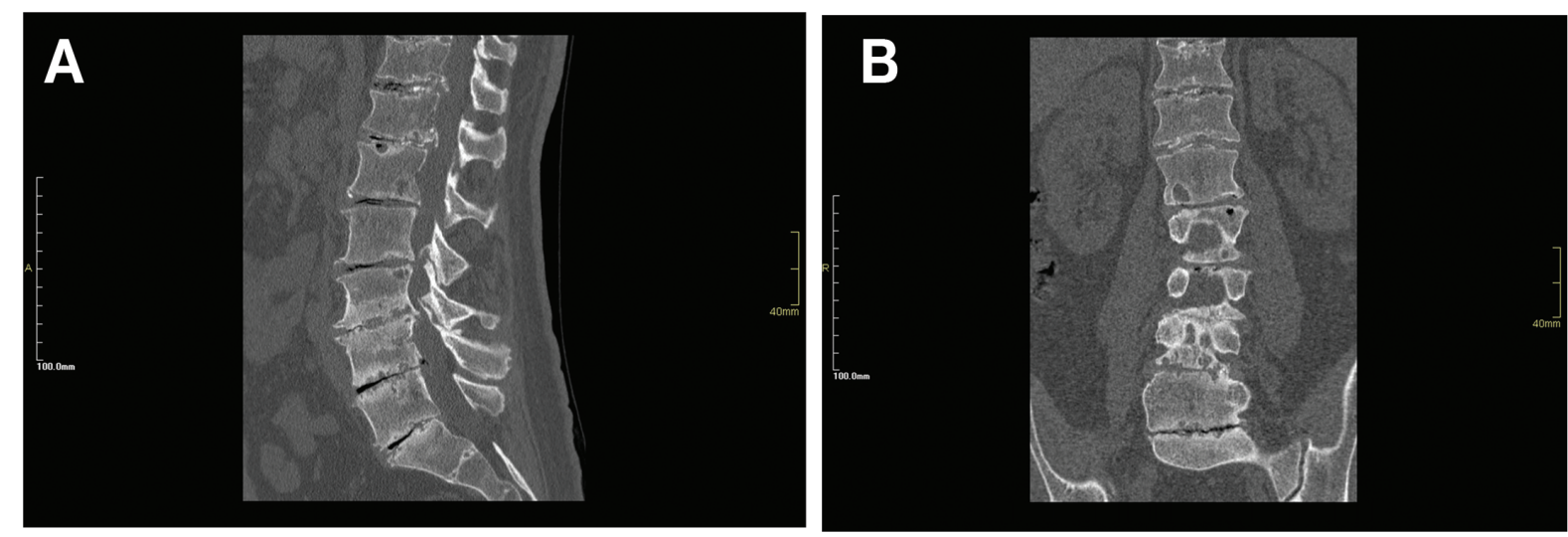

Figure 3. Computed tomographic sagittal (A) and coronal (B) reconstruction of the lumbosacral spine showing diffuse degenerative changes represented by disk space narrowing, subchondral cystic changes and erosions, multilevel endplate sclerosis, marginal sydesmophytosis, and the presence of gas in the degenerated disk spaces (vacuum phenomenon). In addition, evidence of disk calcification is noted in the upper disk space level.

black pigmentation was extensive and reaching the calcaneal tuberosity, extending about $7 \mathrm{~cm}$ from the insertion. The excision involved the entire pigmented segment (Figure 5B) and specimens of the fragmented tendon were sent for histopathology with the suspicion of ochronosis. Tendon advancement was not possible due to the large gap created after the excision $(>5 \mathrm{~cm})$. A decision was taken to graft the flexor hallucis longus (FHL) tendon (Figures 5C-E). The posterior aspect of the calcaneus was involved in the deposition as well; so, it was challenging to find a healthy area to implant the graft. A suitable area $2 \mathrm{~cm}$ anterior to the insertion was prepared after curettage to assure a healthy vascularized bed for the graft. The FHL tendon was forfeited at the Henry knot, and the graft was pulled up through the original incision. With appropriate orientation of the fibers of the FHL tendon, the graft was secured with a conventional screw into the calcaneus. The graft was sufficiently long enough to be sutured over the proximal stump of the Achilles using an absorbable polyglactin 910 suture (Vicryl; Ethicon/Johnson and Johnson). Intraoperatively, a stable plantar and dorsiflexion of the ankle was achieved (Figure 6). Histopathological examination of the resected portion of the tendon revealed a thick fibrosed tendinous tissue with chronic inflammation and focal pigment deposition consistent with the classical features usually seen in alkaptonuria.

The patient was discharged from the hospital 2 days after surgery with a cast fitted in a slight plantar flexion. At 3 weeks, the cast was changed to a walking cast with the 

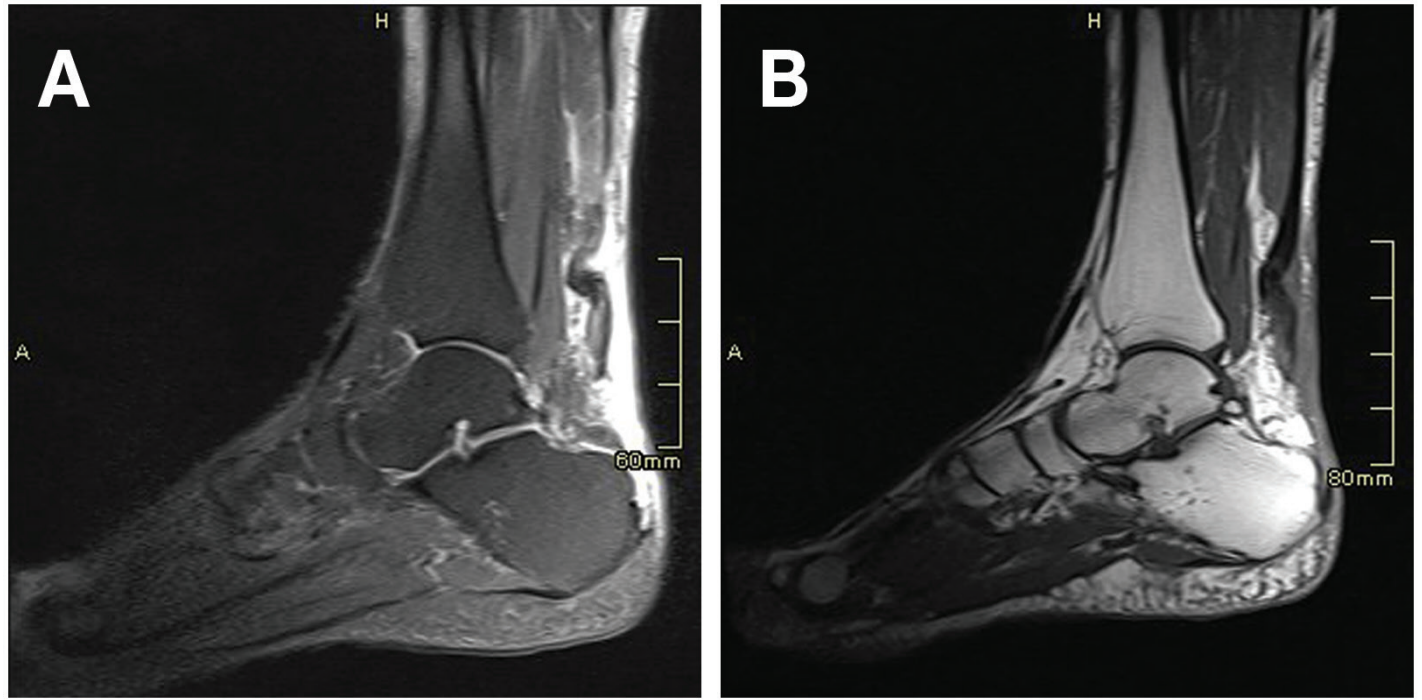

Figure 4. Sagittal fat saturated proton density-weighted (A) and sagittal T1-weighted (B) magnetic resonance images showing an insertional type of Achilles tendon rupture. There is discontinuity and retraction of the Achilles tendon. The tendinous insertion into the calcaneus tuberosity is completely disrupted.
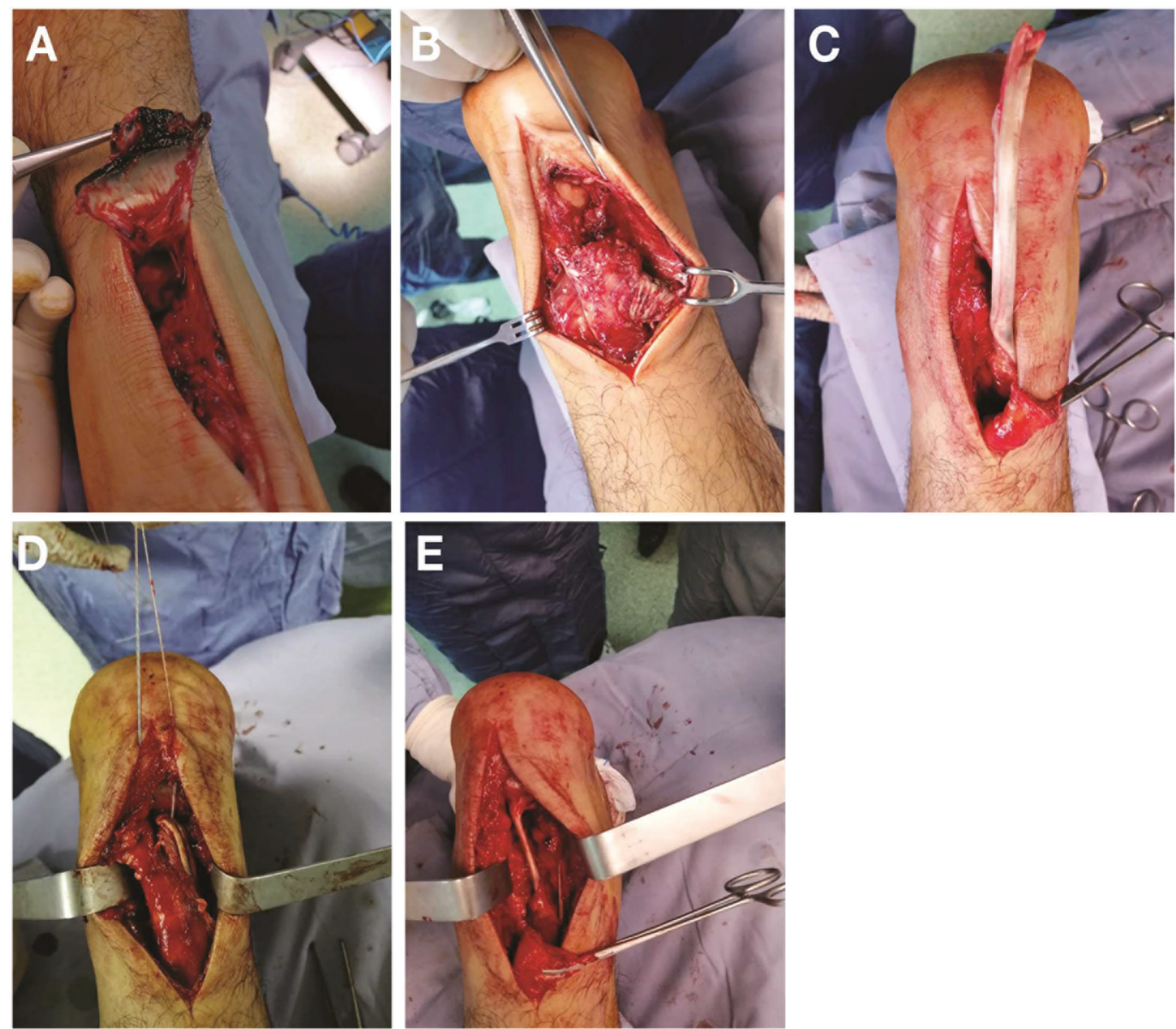

Figure 5. Surgical exploration showing: (A) complete left Achilles tendon rupture at its calcaneal attachment with dark-black pigmentation of the frayed ends, (B) the gap created after removal of the pigmented tissue, (C) the FHL tendon graft, (D) the FHL tendon graft after insertion in the calcaneal tuberosity, and (E) suturing of the proximal stump of the Achilles with the FHL tendon graft using a suture anchor. With documented consent for publication from the patient. FHL, flexor hallucis longus. 

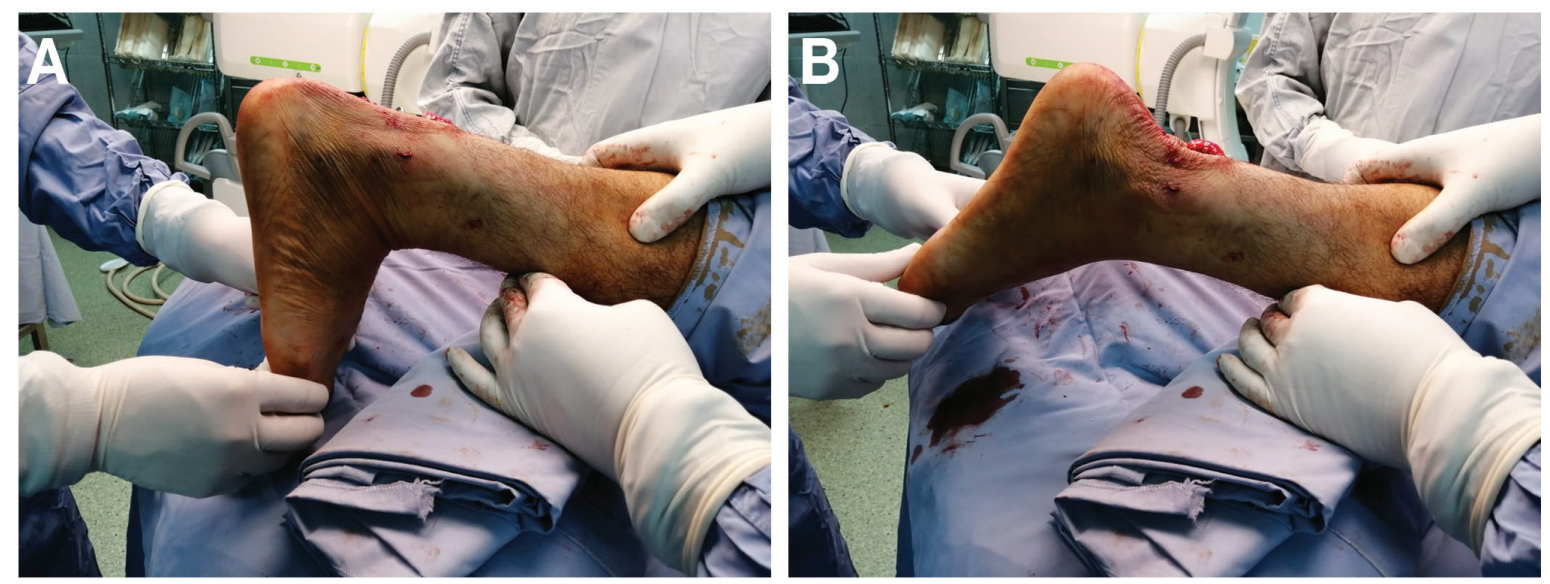

Figure 6. Intraoperative test showing (A) dorsiflexion and (B) plantarflexion of the ankle after repair. With documented consent for publication from the patient.

ankle in a neutral position. At 6 weeks, the cast was removed and the patient started gentle ankle motion physiotherapy. At 10 weeks postoperatively, the patient presented to the clinic walking with the aid of one crutch. Physical examination revealed $0-20^{\circ}$ of plantarflexion and $0-15^{\circ}$ of dorsiflexion. At 6 months' follow-up, our patient presented to the clinic walking without any aid and physical examination revealed a satisfactory range of motion. Written informed consent was obtained from the patient to publish the present case report and associated images, and the CARE reporting guidelines were used when writing the report [18].

\section{Discussion}

Ochronotic osteoarthropathy is the first systemic manifestation in patients with alkaptonuria, starting in their 20 s or 30s. The ochronotic pigment deposited in the bone matrix impairs the bone mineralization process and activates bone resorption $[19,20]$. Additionally, the deposition of the pigmented polymers of HGA in the connective tissues affects the structural integrity of collagen and increases the possibility of tendons to rupture due to their high collagen content [21]. Spontaneous and nontraumatic Achilles tendon rupture due to ochronosis is uncommon and only a few cases from various countries have been reported with their successful surgical treatment. There is no standard method for repairing the tear because the torn ends can be ragged due to extensive degeneration and are not ideal for neat suturing, and most notably, there is a gap length after the excision of the frayed ends. Therefore, several surgical techniques have been utilized for the management of ochronotic Achilles tendon rupture [22], such as direct repair, V-Y advancement, tendon transfer, the Lindholm double facial flap technique, and the Bosworth technique after the removal of the discolored part of the tendon [10,12, 14, 15, 23, 24].

In a case in a 46-year-old Sudanese man, the pigmentation was restricted to a few millimeters from the ruptured surface and the tendon was repaired directly using Vicryl [13]. By contrast, a gap measuring approximately $5 \mathrm{~cm}$ in a 39-year-old Jordanian man was repaired using an inverted V-Y technique to restore the length of the tendon [10]. Baca et al. reported a gap of $6 \mathrm{~cm}$ after debridement and the tendon was repaired using the Lindholm double facial flap technique [12]. However, in a case in a 50-year-old Turkish man with a complete Achilles tendon rupture from its insertion to the calcaneus, approximately $5 \mathrm{~mm}$ of the discolored Achilles tendon was excised and no gap was present after the tendon repair. After debridement, two titanium anchors were fixed at the attachment point on the calcaneus [23].

Here, we report a case of spontaneous rupture of the Achilles tendon at its insertion due to alkaptonuria treated using FHL tendon transfer. The extensive degeneration of the tendon necessitated the removal of a significant portion of the ochronotic tendon, leaving a gap similar to that observed in chronic Achilles tendon rupture. To date, there is still no standard treatment of chronic Achilles tendon rupture with large gaps [25]. However, evaluating the presence or absence of the Achilles tendon stump at the calcaneus and the gap length of rupture gives some indication of the repair technique [26]. When the gap is from $1 \mathrm{~cm}$ to $2 \mathrm{~cm}$ : usually endto-end repair with or without tenodesis augmentation. When 2-5 cm: usually V-Y advancement with or without tenodesis augmentation. For $>5 \mathrm{~cm}$ : autograft or allograft tendon transfer or reconstruction [27]. The FHL tendon is a strong, long tendon, and allows bridging of large gaps [28]. The FHL 
muscle has the same function as the gastrocnemius and soleus; it has the same axis of moving with the Achilles tendon and does not disrupt the normal muscle balance of the ankle [29]. Accordingly, the FHL tendon has been reported to be the most successful for chronic Achilles reconstruction and reconstruction of the degenerated tendon, and it is recommended for all defects $>2 \mathrm{~cm}[30,31]$. In the present case, FHL tendon transfer was considered appropriate for surgical reconstruction with the surgical goal of allowing the patient to regain as much strength as possible. The patient experienced uncomplicated healing with satisfactory functional results. To our knowledge, only one previous case report describes performing long flexor tendon transposition for Achilles tendon rupture due to alkaptonuria [24].

In Achilles tendon repairs, absorbable suture material is associated with a lower incidence of complications compared with nonabsorbable sutures made of material such as polypropylene, which tend to induce foreign-body reaction [32, 33]. Mohammed et al. described the use of Vicryl as an absorbable suture material used to reconstruct a tendon after the resection of ruptured ends [13]. However, some authors reported the use of titanium suture anchors to reattach the tendon to the calcaneus instead of a primary suture fixation to additionally support and reinforce the tendon [23,34]. Ngcelwane et al. reported the use of a running nylon material to suture the paratenon and to reconnect the debrided ends of an ochronotic tendon [35]. Several studies showed that the Achilles tendon healing is promoted by an intact paratenon, the membrane that envelopes the tendon [36]. Asynchronous bilateral rupture of the Achilles tendon has also been reported in some patients with ochronosis $[13,16,34]$. Alkaptonuria is a systemic disease and some cases reported indicate that the incidence of multiple sequential ruptures of various tendons secondary to ochronosis is possible over time [35, 37]. Consequently, prophylactic therapy for alkaptonuric ochronosis is needed to avoid subsequent tendon ruptures. Physicians suggest a lower protein intake particularly of tyrosine-rich food and to take ascorbic acid (vitamin C) at a dose of 0.5-1.0 g/day [12, 35, 38]. Additionally, physical and occupational therapies may be adopted to maintain the flexibility and strength of the muscles and tendons [24]. Nonsteroidal anti-inflammatory drugs for symptomatic treatment as well as glucosamine and chondroitin sulfate supplements, which are considered to slow the degeneration of joint cartilage, have been recommended [23]. The current approach is to use a low dose of nitisinone, which has a role in delaying the disease progression by inhibiting HGA formation and subsequent accumulation in tissues [39, 40]. Recently, 2 clinical trials, SONIA 1 and SONIA 2 approved the efficacy and safety of nitisinone as a therapy for alkaptonuria $[41,42]$.

\section{Conclusions}

The surgical treatment of an Achilles tendon rupture with a large gap is a challenge for most orthopedic surgeons. Ochronotic tendinopathy and ochronotic osteoporosis in patients with alkaptonuria make the treatment even more challenging. Although the technique is technically challenging, this case report provides evidence that FHL tendon transfer is an effective technique for the treatment of spontaneous rupture of a severely ochronotic tendon in a patient with alkaptonuria. Such extensive ochronotic involvement of the Achilles tendon of alkaptonuria has been reported rarely.

Author contributions. All the authors contributed substantially to the conception and design of this study, acquired the data, and interpreted them. NM, AA, MA, and HK drafted the manuscript. SA, OA, and MA revised it critically for important intellectual content. All the authors approved the final version submitted for publication and take responsibility for statements made in the published article.

Acknowledgments. We did not receive any specific grant for this case report from any funding agency in the public, commercial, or not-for-profit sectors.

Conflict of interest statement. The authors have each completed and submitted an International Committee of Medical Journal Editors Uniform Disclosure Form for Potential Conflicts of Interest. None of the authors have any potential or actual conflict of interest to disclose.

Data sharing statement. Data generated or analyzed for the present report are included in this published article. Further details are available from the corresponding author on reasonable request after deidentification from the patient whose data are included in the report. Data were deposited in the HGD mutation database [4] of the Lieden Open Variation Database (LOVD) variant listings with patient ID: AKU_DB_420, patient data (\# 0000720), geographic origin: Al-Karak, Jordan, as submitted by Dr. Nesrin Mwafi. Available at the following URL: http://hgddatabase.cvtisr.sk/variants. php?action=search_unique\&select_db=HGD

\section{References}

[1] Zatkova A, Ranganath L, Kadasi L. Alkaptonuria: current perspectives. Appl Clin Genet. 2020; 13:37-47.

[2] Ranganath LR, Norman BP, Gallagher JA. Ochronotic pigmentation is caused by homogentisic acid and is the key event 
in alkaptonuria leading to the destructive consequences of the disease - a review. J Inherit Metab Dis. 2019; 42:776-92.

[3] Ventura-Ríos L, Hernández-Díaz C, Gutiérrez-Pérez L, BernalGonzález A, Pichardo-Bahena R, Cedeño-Garcidueñas AL, Pineda C. Ochronotic arthropathy as a paradigm of metabolically induced degenerative joint disease. A case-based review. Clin Rheumatol. 2016; 35:1389-95.

[4] Zatkova A, Sedlackova T, Radvansky J, Polakova H, Nemethova M, Aquaron R, et al. Identification of eleven novel homogentisate 1,2 dioxygenase (HGD) variants in alkaptonuria (AKU) patients and establishment of a novel LOVD based HGD mutation database. JIMD Rep. 2012; 4:55-65.

[5] Al-sbou M, Mwafi N. Nine cases of Alkaptonuria in one family in southern Jordan. Rheumatol Int. 2012; 32:621-5.

[6] Mwafi NR, Ali DA, Khalil RW, Alsbou' IN, Saraireh AM. Novel R225C variant identified in the $H G D$ gene in Jordanian patients with alkaptonuria. AIMS Mol Sci. 2021; 8:60-75.

[7] Wu K, Bauer E, Myung G, Fang MA. Musculoskeletal manifestations of alkaptonuria: a case report and literature review. Eur J Rheumatol. 2019; 6:98-101.

[8] Kural C, Çetınus EM, Kural A, Uğraş AA, Kaya I. Diz okronotik artropatisi ve artroskopik bulguları [Knee ochronotic arthropathy and arthroscopic findings]. Acta Orthop Traumatol Turc. 2009; 43:67-71. [in Turkish, English abstract and author translation]

[9] Al-Shagahin HM, Mwafi M, Khasawneh M, Al Zubi K, Alsbou M. Ear, nose, and throat manifestations of alkaptonuria patients from Jordan. Indian J Otol. 2019; 25:109-13.

[10] Alajoulin OA, Alsbou MS, Ja’afreh SO, Kalbouneh HM. Spontaneous Achilles tendon rupture in alkaptonuria. Saudi Med J. 2015; 36:1486-9. [Arabic abstract]

[11] Wolff F, Biaou I, Koopmansch C, Vanden Bossche M, Pozdzik A, Roumeguère T, Cotton F. Renal and prostate stones composition in alkaptonuria: a case report. Clin Nephrol. 2015; 84:339-43.

[12] Baca E, Kural A, Ziroglu N, Kural C. Alkaptonuria: spontaneous Achilles tendon rupture: case report [Alkaptonüri: spontan Aşil tendonu rüptürü: olgu sunumu]. Eklem Hastalik Cerrahisi. 2019; 30:325-8. [Turkish abstract]

[13] Mohammed OB, Idris SI, Shalayel MH. Spontaneous Achilles tendon rupture in alkaptonuria. Sudan J Med Sci. 2011; 6:143-5.

[14] Prakash V, Boston DA, Packer GJ, Raza SA. Rupture of a black tendon: ochronotic tendo-Achilles rupture. The Foot. 2003; 13:49-50.

[15] Mahesh DV, Gunnaiah, Deepak CD, Chandar V. A rare case of alkaptonuria - Achilles tendon rupture. Int J Health Sci Res. 2014; 4(5):323-7.

[16] Shetty SK, Raj Sankar NR, Nirmal Babu P, Mathias LJ, Bhat SP, Rai R. Spontaneous Achilles tendon rupture - a case of ochronosis. Nitte Univ J Health Sci. 2013; 3:113-5.

[17] Phornphutkul C, Introne WJ, Perry MB, Bernardini I, Murphey MD, Fitzpatrick DL, et al. Natural history of alkaptonuria. N Engl J Med. 2002; 347(26):2111-21.

[18] Gagnier JJ, Kienle G, Altman DG, Moher D, Sox H, Riley D, et al. The CARE guidelines: consensus-based clinical case reporting guideline development. Glob Adv Health Med. 2013; 2:38-43.

[19] Taylor AM, Hsueh M-F, Ranganath LR, Gallagher JA, Dillon JP, Huebner JL, et al. Cartilage biomarkers in the osteoarthropathy of alkaptonuria reveal low turnover and accelerated ageing. Rheumatology (Oxford). 2017; 56:156-64.
[20] Aldossary SA, Alsalem M, Kalbouneh H, Haddad M, Azab B, $\mathrm{Al}-\mathrm{Shboul} \mathrm{O}$, et al. The role of transient receptor potential vanilloid receptor 1 and peroxisome proliferator-activated receptors- $\alpha$ in mediating the antinociceptive effects of palmitoylethanolamine in rats. Neuroreport. 2019; 30:32-7.

[21] Grosicka A, Kucharz EJ. Alkaptonuria. Wiad Lek. 2009; 62:197-203. [Polish abstract]

[22] Khiami F, Di Schino M, Sariali E, Cao D, Rolland E, Catonné Y. Treatment of chronic Achilles tendon rupture by shortening suture and free sural triceps aponeurosis graft. Orthop Traumatol Surg Res. 2013; 99:585-91.

[23] Tanoğlu O, Arıcan G, Özmeriç A, Alemdaroğlu KB, Çaydere M. Calcaneal avulsion of an ochronotic Achilles tendon: a case report. J Foot Ankle Surg. 2018; 57:179-83.

[24] Jiang L, Cao L, Fang J, Yu X, Dai X, Miao X. Ochronotic arthritis and ochronotic Achilles tendon rupture in alkaptonuria: a 6 years follow-up case report in China. Medicine (Baltimore). 2019; 98:e16837. doi: 10.1097/MD.0000000000016837

[25] Ahmad J, Jones K, Raikin SM. Treatment of chronic Achilles tendon ruptures with large defects. Foot Ankle Spec. 2016; 9:400-8.

[26] Lin Y, Yang L, Yin L, Duan X. Surgical strategy for the chronic Achilles tendon rupture. BioMed Res Int. 2016; 2016:1416971. doi: 10.1155/2016/1416971

[27] Molloy A, Myerson M. Chapter 112. Chronic Achilles tendon ruptures using allograft reconstruction. In: Section V. Sportsrelated procedures for ankle and hindfoot. In: Easley M, Wiesel S, editors. Operative techniques in foot and ankle surgery. Philadelphia: Lippincott Williams \& Wilkins; 2011, p. 933-6.

[28] Maffulli N, Via AG, Oliva F. Chronic Achilles tendon rupture. Open Orthop J. 2017; 11(Suppl 4, M6):660-9.

[29] Rahm S, Spross C, Gerber F, Farshad M, Buck FM, Espinosa $\mathrm{N}$. Operative treatment of chronic irreparable Achilles tendon ruptures with large flexor hallucis longus tendon transfers. Foot Ankle Int. 2013; 34:1100-10.

[30] Lin JL. Tendon transfers for Achilles reconstruction. Foot Ankle Clin. 2009; 14:729-44.

[31] Den Hartog BD. Surgical strategies: delayed diagnosis or neglected Achilles' tendon ruptures. Foot Ankle Int. 2008; 29:456-63.

[32] Park JH, Chun D, Lee SH, Cho JH. A comparative evaluation of absorbable and nonabsorbable sutures for open repair of Achilles tendon rupture - a pilot study. Korean J Phys Anthropol. 2017; 30:39-46.

[33] Baig MN, Yousaf I, Galbraith JG, Din R. Absorbable Polydioxanone (PDS) suture provides fewer wound complications than polyester (ethibond) suture in acute Tendo-Achilles rupture repair. Ir Med J. 2017; 110:566. Available from: http://hdl.handle.net/10147/622537

[34] Awad A, Abdelmaged H, Ahmed H, Alnaser A, Mukhtar S. Bilateral asynchronous spontaneous Achilles tendon rupture in alkaptonuria: a case report. Sch J App Med Sci. 2020; 8 : 2685-9.

[35] Ngcelwane M, Mandaba M, Bam T. Multiple tendon ruptures in ochronosis: case report and review of prophylactic therapy. SA Orthop J. 2013; 12:55-7.

[36] Müller SA, Evans CH, Heisterbach PE, Majewski M. The role of the paratenon in Achilles tendon healing: a study in rats. Am J Sports Med. 2018; 46:1214-9. 
[37] Kumar EGM, Kumar GMY. Sequential tendon ruptures in ochronosis: case report. Int J Res Orthop. 2017; 3:1235-8.

[38] Braconi D, Laschi M, Taylor AM, Bernardini G, Spreafico A Tinti L, et al. Proteomic and redox-proteomic evaluation of homogentisic acid and ascorbic acid effects on human articular chondrocytes. J Cell Biochem. 2010; 111:922-32.

[39] Keenan CM, Preston AJ, Sutherland H, Wilson PJ, Psarelli EE, Cox TF, et al. Nitisinone arrests but does not reverse ochronosis in alkaptonuric mice. In: Zschocke J, Baumgartner M, Morava E, Patterson M, Rahman S, Peters V, editors. JIMD Reports, volume 24. Berlin, Heidelberg: Springer; 2015, p. 45-50.

doi: 10.1007/8904_2015_437

[40] Ranganath LR, Khedr M, Milan AM, Davison AS, Hughes AT, Usher JL, et al. Nitisinone arrests ochronosis and decreases rate of progression of alkaptonuria: evaluation of the effect of nitisinone in the United Kingdom National Alkaptonuria Centre. Mol Genet Metab. 2018; 125:127-34.

[41] Ranganath LR, Milan AM, Hughes AT, Dutton JJ, Fitzgerald $\mathrm{R}$, Briggs MC, et al. Suitability Of Nitisinone In Alkaptonuria 1 (SONIA 1): an international, multicentre, randomised, open-label, no-treatment controlled, parallel-group, dose-response study to investigate the effect of once daily nitisinone on 24-h urinary homogentisic acid excretion in patients with alkaptonuria after 4 weeks of treatment. Ann Rheum Dis. 2016; 75:362-7.

[42] Ranganath LR, Psarelli EE, Arnoux J-B, Braconi D, Briggs M, Bröijersén A, et al. Efficacy and safety of once-daily nitisinone for patients with alkaptonuria (SONIA 2): an international, multicentre, open-label, randomised controlled trial. Lancet Diabetes Endocrinol. 2020; 8:762-72. 